\title{
NUMERICAL EVIDENCE FOR CUTOFFS IN CHAOTIC MICROFLUIDIC MIXING
}

\author{
Tzu-Chen Liang \\ Department of Aeronautics and Astronautics \\ Stanford University \\ Stanford, California 94305 \\ Email: tzuchen@stanford.edu
}

\author{
Matthew West \\ Department of Mechanical Science and Engineering \\ University of Illinois at Urbana-Champaign \\ Urbana, Illinois, 61801 \\ Email: mwest@illinois.edu
}

\begin{abstract}
Chaotic mixing strategies produce high mixing rates in microfluidic channels and other applications. In prior numerical and experimental work the variance of a tracer field in a chaotic mixer has been observed to decay rapidly after an initial slower transient. We relate this to the cutoff phenomenon observed in finite Markov chains and provide numerical evidence to suggest that chaotic mixing indeed exhibits cutoff. We provide results for a herringbone passive microfluidic mixer and the Standard Map.
\end{abstract}

\section{INTRODUCTION}

The question of how chaotic advection mixes a passive scalar function has attracted much research effort in recent years [1]. The main issues in this field are: how to measure the thoroughness of the mixing, how the mixing process changes qualitatively and quantitatively when the diffusion is close to zero, and how to enhance the overall mixing process by designing the map which produces chaotic advection. Unfortunately, we have only partial understanding for most of these topics. In spite of the fact that the detailed mechanism of mixing is unclear, nontrivial mixing processes have been observed in experiments [2] and can be simulated by large-scale computations [3].

A widely observed phenomenon in the chaotic mixing process when small diffusion exists is the two or three-stage transition [4-6]. The map does not mix the scalar function with a constant rate in general. When the variance of the scalar function is measured during the mixing process, one can in general observe a relatively flat decay initially, followed by a super-exponential change, and then finally it tends to an exponential decay. We are interested in when these transitions happen, why they happen, and how to predict the slope of the exponential region. A good review and physical interpretation can be found in [7].
Thiffeault and Childress [4] study these properties for a modified Arnold's cat map. Analytical formulas are given to predict the transitions as well as the slopes. Because the linear part of this map has an eigenvalue 2.618, which stretches very fast, and the chaotic part is relatively small, the three phases are separated clearly. The same analytical procedure cannot be applied to, for example, the Standard Map, although the only difference between the Standard Map and the modified Arnold's cat map is in the linear part.

As for the exponential decay part, there is still debate about whether the decay rate goes to zero in the zero diffusivity limit or whether it tends to a constant independent of the diffusion [3,7]. Theoretical analysis shows both of these possibilities can occur for different chaotic flows [8].

Difficulties typically arise in studying the above problems numerically, because the small diffusion usually means that fine grids are required in the solution of the advection-diffusion equation or the simulation of the map. Some studies and numerical results conclude that a proportional relation exists between the stationary decay rate and the diffusion [9]. However, this is only true for certain diffusion ranges.

Our goal in this paper is to relate the chaotic mixing process to the well-known cutoff phenomenon in finite Markov Chain studies (see [10] and references therein). We begin with a numerical simulation of a chaotic mixing channel, measuring mixing of two colored liquids by the color variance of channel crosssections. The simulation shows that when we increase the Péclet number, the mixing trajectory presents a cutoff. The underlying physical mechanism is then explained using advection of a sinusoidal function under the Baker's Map. To support the chaotic mixing channel example, a very high resolution numerical simulation of the Standard Map is then presented to show that in the near-zero diffusion limit it does present a cutoff. 


\section{BACKGROUND}

\section{The measure space and operators}

We work on the probability space $(X, \mathcal{A}, \mu)$ for $X$ a subset of $\mathbb{R}^{n}$. We take $S: X \rightarrow X$ to be a transformation (or map) that is non-singular and measurable. We choose $\mu$ to be the Borel measure. In the measure space $(X, \mathcal{A}, \mu)$ we define the following operators.

Definition 1. (Perron-Frobenius operator) The PerronFrobenius operator $P: L^{1}(X) \rightarrow L^{1}(X)$ associated with $S$ satisfies

$$
\int_{A}(P \omega)(x) \mu(d x)=\int_{S^{-1}(A)} \omega(x) \mu(d x)
$$

for every $\omega \in L^{1}(X)$ and $A \in \mathcal{A}$.

The Perron-Frobenius operator is linear. Because of our choice of measure space, the Perron-Frobenius operator can be interpreted as a map that evolves probability density functions. Also, suppose that $\bar{\omega}$ is an invariant measure of $S$, so that $\bar{\omega}\left(S^{-1}(A)\right)=$ $\bar{\omega}(A)$ for all $A \in \mathcal{A}$. Then we have $P \bar{\omega}=\bar{\omega}$ (omitting $x$ ).

Definition 2. (Koopman operator) Let $f \in L^{\infty}(X)$. The operator $U: L^{\infty}(X) \rightarrow L^{\infty}(X)$ defined by $U f(x)=f(S(x))$ is called the Koopman operator associated with $S$.

The Koopman operator is adjoint to Perron-Frobenius operator, which we write as $U=P^{*}$.

In the measure space $(X, \mathcal{A}, \mu)$, where $\mu$ is the Borel measure, let $P_{S}$ and $U_{S}$ be the Perron-Frobenius and the Koopman operators of an invertible map $S$. We have the following relations:

\begin{tabular}{l|ll} 
& forward in time & backward in time \\
\hline probability density & $P_{S}$ & $P_{S^{-1}}$ \\
scalar function & $U_{S^{-1}}=P_{S^{-1}}^{*}$ & $U_{S}=P_{S}^{*}$
\end{tabular}

Our goal is to simulate how a scalar function is advected by a chaotic map forward in time. From the above table, it is clear we should use the operator $U_{S^{-1}}$. For a given initial function $f^{0}(x)$, one has, $f^{k+1}=U_{S^{-1}} f^{k}$, for all $k$.

\section{Numerical Strategy}

Numerically, an approximation of $U_{S^{-1}}$ is used. For a map $S: X \rightarrow X$, we discretize $X$ into regular square grids with size $h$. The grids are numbered as $a_{1}, a_{2}, . ., a_{n}$. We first define a map (an observer) $g_{n}: f(x) \mapsto f_{n}$ such that

$$
\left(f_{n}\right)_{i}=\left(g_{n}(f(x))\right)_{i}=\int_{a_{i}} f(x) \mu(d x), \text { for } i=1 \text { to } n .
$$

This $g_{n}$ maps the scalar function we are interested into a finite length vector in $\mathbb{R}^{n}$, and thus we only need a linear operator $B_{n}$ :
$\mathbb{R}^{n} \rightarrow \mathbb{R}^{n}$ to approximate $U_{S^{-1}}$, and thus to evolve the function in the reduced space $\mathbb{R}^{n}$. The $B_{n}$ we use in our numerical simulation is obtained in a very simple way: let $\mathbf{x}_{i}=\left(x_{1 i}, x_{2 i}\right)$ be the center of grid $a_{i}$, then define a matrix $B_{n}$ as,

$$
\left(B_{n}\right)_{i j}= \begin{cases}\frac{1}{4} & , \text { if } S\left(x_{1 j} \pm \frac{h}{2}, x_{2 j} \pm \frac{h}{2}\right) \in a_{i} \\ 0 & , \text { otherwise }\end{cases}
$$

The matrix $B_{n}$ has only 4 non-zeros in each row. For a given $f^{0}$, we can thus approximate the evolution by $f_{n}^{k+1}=B_{n} f_{n}^{k}$.

This approach is similar to the lattice method $[3,11]$. We stress that there exist better approximations of $U_{S^{-1}}$ which minimize the difference between $f_{n}^{k}$ and $g_{n}\left(f^{k}\right)$ [12] by applying optimal model reduction. However, our simple numerical strategy allows us to go up to very large $n$ and to simulate the system with very small numerical diffusion. During the simulation, the matrix $B_{n}$ is never explicitly formed and we need only store a length $n$ state vector, ensuring system evolution has cost of order $n$.

Using the above numerical strategy, we can evolve a function or a probability distribution by the map with some small numerical diffusion. The effect of numerical diffusion is similar to physical diffusion on large scales, but their behavior can be quite different on small scales. To simulate the physical diffusion correctly, we need to simulate the map with higher resolution with some additional physical diffusion added. The additional diffusion can be added in either spatial or frequency domains. In the spatial domain, we adopt the method of adding a smoothing step used in [3],

$$
f_{(p, q)}^{k+1}=\sum_{|r|,|s| \leq 2} C_{|r|} C_{|s|} f_{(p, q)}^{k}
$$

with $C_{0}=1 / 8, C_{1}=1 / 4$, and $C_{2}=3 / 16$ and $(p, q)$ is the 2-D index of a grid. This creates a large scale diffusion $D \approx h^{2}$, which is several times larger than the numerical diffusion [3]. We use a smoothing operator $M_{n}$ and $f^{k+1}=M_{n}\left(f^{k}\right)$ to denote the above smoothing step and define $\bar{B}_{n}=M_{n} \circ B_{n}$.

Alternatively, in frequency domain a two dimensional FFT/IFFT with a wave-number dependent scaling can be applied to simulate physical diffusion. This procedure is denoted by an operator $F_{n}, f^{k+1}=F_{n}\left(f^{k}\right)$ and $\hat{B}_{n}=F_{n} \circ B_{n}$. Note that the FFT/IFFT scheme is much more expensive when $n$ is large.

\section{Notion of a cutoff}

In some Markov Chains, certain probability distributions converge to an equilibrium via a sharp transition, which becomes sharper for larger chains. This phenomenon is referred to as cutoff in the finite Markov chain literature [10]. Here we extend the usual definition slightly to accommodate converge to non-zero distance values. 
To any finite set $\Omega$ and any pair of probability measures $\omega$, $\bar{\omega}$ on $\Omega$ we associate a real number $d(\omega, \bar{\omega})$ such that

$$
\begin{aligned}
d(\omega, \bar{\omega}) & \in[0,1] \\
d(\omega, \bar{\omega}) & =0 \text { if and only if } \bar{\omega}=\omega \\
\max _{\Omega, \omega, \bar{\omega}} d(\omega, \bar{\omega}) & =M_{d} .
\end{aligned}
$$

Note that $d$ need not satisfy the triangle inequality and so is not a metric.

Consider a sequence of finite probability spaces $\left(\Omega_{n}\right)$ for $n=1,2, \ldots$. We think of $n$ as the size of the space. Each space is equipped with a probability measure $\bar{\omega}_{n}$ which we think of as the unique invariant measure of a Markov chain on $\Omega_{n}$. For each $n$ we now take a sequence of probability measures $\omega_{n}^{k}$ for $k=0,1,2, \ldots$ such that $\lim _{k \rightarrow \infty} d\left(\omega_{n}, \bar{\omega}_{n}\right)=0$. The $\omega_{n}^{k}$ should be thought of as an initial condition $\omega_{n}^{0}$ and then iterates of the distribution under the evolution of a Markov chain.

Definition 3 (Cutoff). Take a family $\left(\Omega_{n}, \bar{\omega}_{n},\left(\omega_{n}^{k}\right)_{k=0}^{\infty}\right)_{n=1}^{\infty}$ of finite probability spaces $\Omega_{n}$ and probability measures $\bar{\omega}_{n}$ and $\omega_{n}^{k}$. This family presents a d-cutoff if there exists a sequence $\left(t_{n}\right)_{n=1}^{\infty}$ of positive reals such that, for any $\varepsilon \in(0,1)$,

$$
\begin{aligned}
& \lim _{n \rightarrow \infty} d\left(\omega_{n}^{k_{n}}, \bar{\omega}_{n}\right)=m \text { if } k_{n}>(1+\varepsilon) t_{n} \\
& \lim _{n \rightarrow \infty} d\left(\omega_{n}^{k_{n}}, \bar{\omega}_{n}\right)=M \text { if } k_{n}<(1-\varepsilon) t_{n}
\end{aligned}
$$

This definition is taken from [10] with the change that $m$ and $M$ are 0 and $M_{d}$ in the original. The reason for this modification will be clear when we present the results of Standard Map simulation.

The definition of cutoff implies that the change of $d\left(\omega_{n}^{k}, \bar{\omega}_{n}\right)$ from $M$ to $m$ happens ever more rapidly as $n$ increases, but only in relation to the cutoff times $t_{n}$. We can think of this as rescaling the each trajectory $\left(\omega_{n}^{k}\right)_{k=0}^{\infty}$ in time by $t_{n}$ and seeing cutoff as the limit of these rescaled trajectories to a step function. We give an example of cutoff phenomenon:

Example 1. Random walk on an n-dimensional hypercube (Diaconis [13]) A particle starts at the origin and moves to one of its nearest neighbors (or stays fixed) with equal probability at each step. The problem can be formulated as a Markov Chain with $2^{n}$ states and the invariant distribution $\bar{\omega}$ is uniform. The $\left|\omega_{n}^{k}-\bar{\omega}\right|_{T V}$ (Total Variation Distance) versus iteration plot with different $n$ is shown in Figure 1.

\section{How to recognize a cutoff?}

To prove the existence of a cutoff is in general very hard, relying on special features of the sequence of systems. In this article we merely provide numerical evidence suggesting that cutoffs occur. The way we determine whether a sequence of
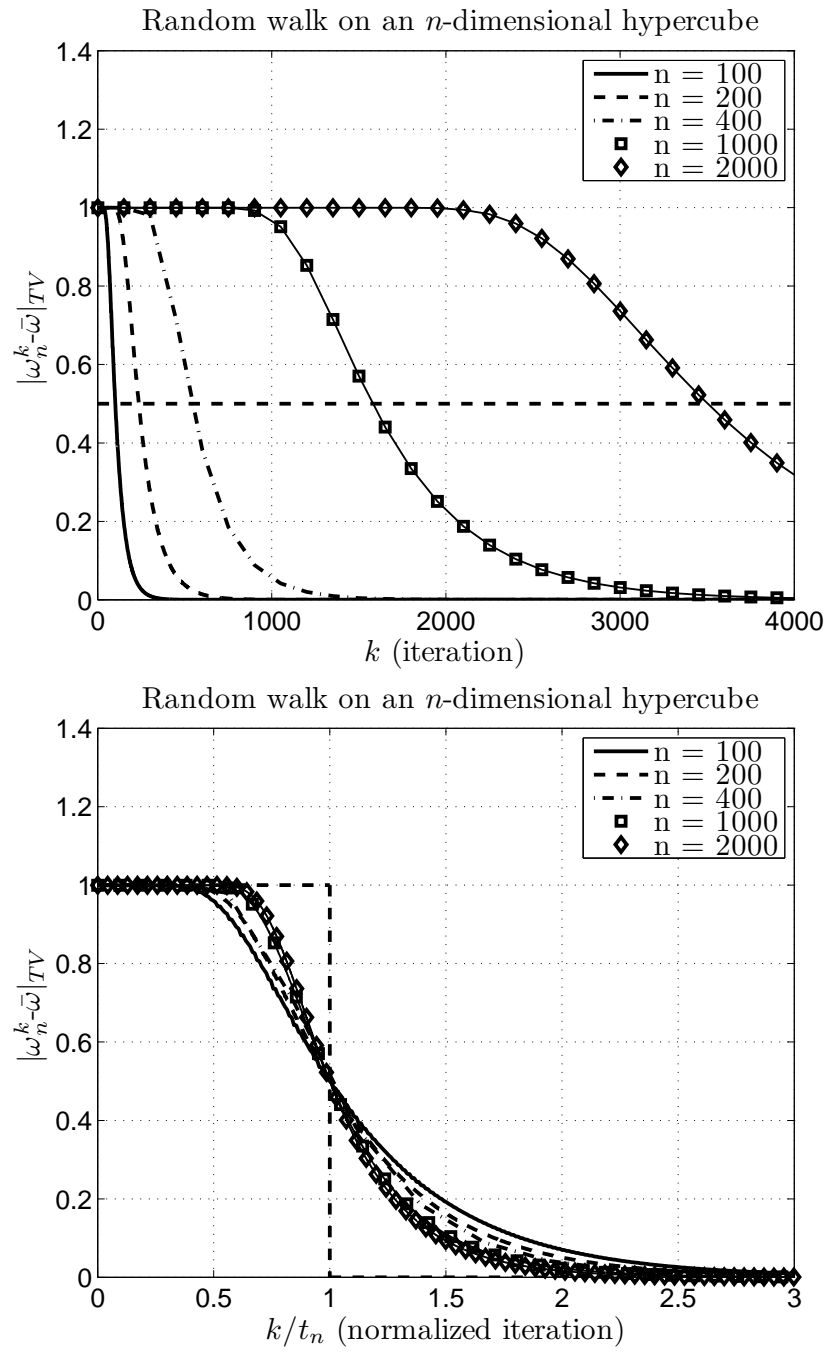

Figure 1. THE UPPER FIGURE SHOWS THE EVOLUTION OF A MEASURE TO THE INVARIANT MEASURE FOR A RANDOM WALK ON AN $n$-DIMENSIONAL HYPERCUBE. WHEN $n$ INCREASES, THE DISTANCE STAYS CLOSE TO 1 FOR LONGER BEFORE IT DROPS TO ZERO. THE BOTTOM FIGURE HAS TRAJECTORIES RESCALED IN $k$ TO SHOW CUTOFF AS A LIMIT TO A STEP FUNCTION.

simulation results presents a cutoff or not is through the definition. We first define a cutoff time $t_{n}$, usually the number of iterations required for the trajectory indexed by $n$ to pass through $\left(M_{\star}+m_{\star}\right) / 2$, where $\star$ indicates the operator used in the simulation, and then all the trajectories are rescaled by their $t_{n}$ on the iteration axis. If the normalized plot shows the tendency to converge to the function:

$$
\beta_{\star_{\infty}}(x)= \begin{cases}M_{\star} & , \text { if } x<1, \\ m_{\star} & , \text { otherwise }\end{cases}
$$

then this suggests that the sequence of simulations presents a cutoff. To determine this, we define the interpolating functions for 
the trajectories in the normalized plots to be $\beta_{\star_{n}}(x)$, where $x$ represents the normalized iteration, and then define the distance between $\beta_{\star_{n}}(x)$ and $\beta_{\star_{\infty}}(x)$ to be $\Delta_{\star}^{\ell}$, given by

$$
\Delta_{\star}^{\ell}=\int_{0}^{\ell}\left|\beta_{\star_{\infty}}(x)-\beta_{\star_{n}}(x)\right| d x
$$

We calculate $\Delta_{\star}^{3}$ for all $\beta_{\star}(x)$, and plot them versus $t_{n}$, thus indicating whether this sequence of simulations is likely to present a cutoff.

An important quantity in chaotic mixing is the variance of the function, and in the study of cutoff phenomenon, total variation distance is commonly used. There is no difficulty to set the distance function $d(\omega, \bar{\omega})$ to be the 2-norm as the following:

$$
d\left(\omega_{n}, \bar{\omega}_{n}\right)=\left(\sum_{i=1}^{n}\left(\frac{\left(\omega_{n}\right)_{i}}{\left(\bar{\omega}_{n}\right)_{i}}-1\right)^{2}\left(\bar{\omega}_{n}\right)_{i}\right)^{1 / 2}
$$

This corresponds to the study of $L_{2}$ cutoff in the cutoff terminology.

It is clear that the maximal value of the 2-norm distance is $\infty$, so we need to set $M_{d}=\infty$ (in stead of $M_{d}=1$ for total variation distance). In the original cutoff definition, $(M, m)$ always equals $\left(M_{d}, 0\right)$, but in our examples we will set $M$ to have a finite value ( 0.5 in the mixing channel case and 1 in the Standard Map case) which does not maximize the distance function.

\section{THE MICROFLUIDIC MIXING CHANNEL}

Microfluidic systems control and manipulate liquids in microliter or nanoliter amounts. One of the challenges in microfluidics is the design of mixing channels, whose objective is to thoroughly mix two or more different liquids. Passive mixers, such as discussed in this paper, do not actively change system geometry to mix the fluids. This reliance on fixed system design has advantages in manufacturing simplicity and price.

Active mixing, while not investigated here, shows very promising results by using components like micro-pumps to stir the flow [14], and also allows the use of feedback strategies developed for boundary-controlled Navier-Stokes systems [15-19].

A microfluidic mixing channel typically has cross-section dimension $\ell \sim 100 \mu m$, and Reynolds number $\operatorname{Re}=U \ell / \mathrm{v}$ is less than 100 [20] ( $U$ is the average velocity of the liquid and $v$ is the kinematic viscosity of the fluid). Fluid flow on this scale is highly laminar and the mixing of materials between streams is purely diffusive. The dimensionless number that controls the length of the channel required for mixing is the Péclet number $(\mathrm{Pe}=U \ell / D$ where $D$ is the molecular diffusivity). For a pressure-driven mixing channel the mixing length can be expected to grow linearly with $P e$ and is usually much more than $1 \mathrm{~cm}$. Hence various designs are proposed to stir the flow inside the channel and produce transverse velocities to enhance the mixing $[20,21]$.
Stroock, et. al [20] proposed a staggered herringbone mixer which is composed of two sequential regions of ridges; the direction of asymmetry of the herringbones switches with respect to the center-line of the channel from one region to the next. The herringbone structure is fabricated with two steps of photolithography and is located on the floor of the poly channel. Experiments show that the length of the channel required for mixing grows only logarithmically with Pe. The goal of the herringbone structure is to produce transverse flows, basically one large and one small vortex, and we further optimize in the structure [22] by using the techniques of topology optimization. The optimized half-cycle structure is shown in Figure 2, where $\left(\ell_{x}, \ell_{y}, \ell_{z}\right)=(0.06,0.01,0.02) \mathrm{cm}$. The same pattern repeats four times in the half-cycle structure. One full-cycle is composed of two half-cycle channels which arrange as in Strook's design.

We stimulate the mixing process of the proposed mixing channel by first solving the velocity field for one full-cycle of the mixing channel, and then defining an inlet-outlet flow map by integrating the streamlines. Once the flow map is obtained, we can apply our numerical strategy to simulate the mixing process. The actual mixing process happens in between the streamlines and is the solution of an advection-diffusion equation. We approximate this complicated process by the simple model outlined above, and lump all diffusion inside the mixing channel into an FFT/IFFT diffusion operator $F_{n}$. Thus the operator to perform this simulation is $\hat{B}$.

In Figure 3, the optimized mixing channel is used to perform the simulation with different Pe. We adjust Pe by changing the FFT/IFFT diffusivity between each full-cycle $(0.12 \mathrm{~cm})$. The trajectories have the same tendency as the experiment results in the Figure 3(D) in [20]. Define mixing length $\left(x_{90}\right)$ as the channel length required for the standard deviation to drop to 0.05 (shown by a dashed line in figure 3 ). The mixing length grows linearly with $\log (\mathrm{Pe})$, which also matches the experiments in [20].

Let $n=$ Pe. Define $t_{n}$ as the number of iterations required for each of the trajectories to pass through 0.25 . The normalized plot is shown in the first plot of Figure 4. One can see a cutoff clearly forming. The cutoff time versus Pe trajectory is shown in the second plot of Figure 4. Just like $x_{90}$, the cutoff time grows linearly with Pe. The $\Delta_{\hat{B}}^{3}$ versus Pe plot is shown in the last plot of Figure 4 . The decreasing trajectory implies that the normalized trajectories is likely to form a cutoff.

Figure 5 shows cross-sectional plots of two of the simulations in Figure 3, where Pe is $1.2 \times 10^{6}$ and $1.2 \times 10^{9}$, respectively. The first four plots of each case show the cross-section at the end of the 1st to the 4th cycles, and the last plot for each case shows the cross-section at the end of the 9th cycle. From this comparison one can clearly see how the chaotic mixing protocol helps color mixing even when diffusion is very small.

More details about this simulation can be found in [22]. In this article, we are more interested in relating the trajectories shown in Figure 3 to the cutoff phenomenon. However, large Pe means fine grids and thus many streamlines need to be calculated. Further increasing Pe and observing a clearer cutoff is 


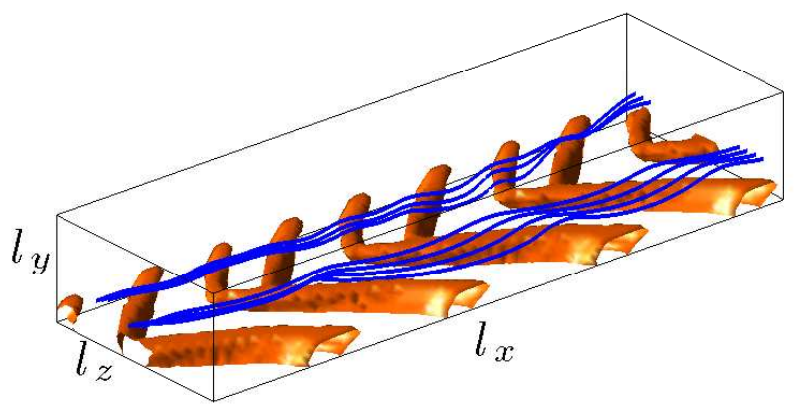

Figure 2. THE OPTIMIZED CHANNEL STRUCTURE TO PRODUCE ONE BIG AND ONE SMALL VORTEX.

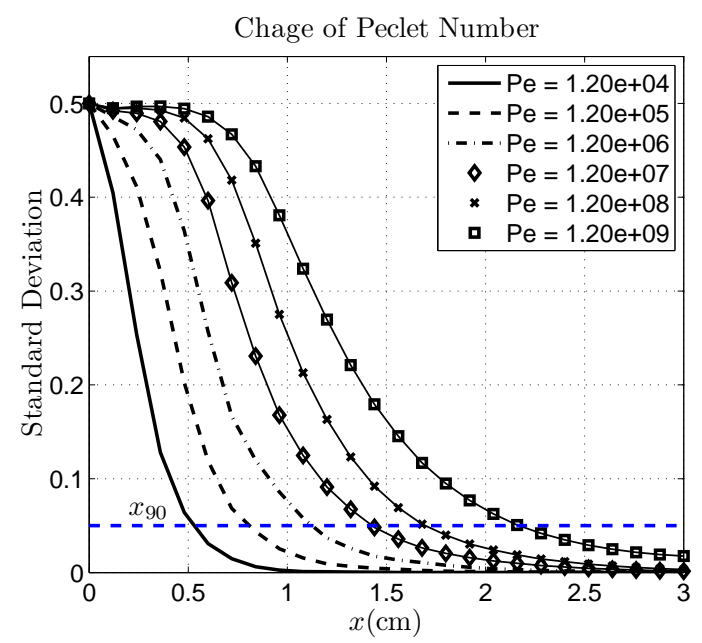

Figure 3. MICROFLUIDIC MIXING AS A FUNCTION OF CHANNEL LENGTH, FOR VARYING Pe, USING THE OPTIMIZED HERRINGBONE STRUCTURED CHANNEL. THE MIXING TRAJECTORIES STAYS ALMOST 0.5 FOR A LONGER DISTANCE WHEN Pe IS LARGE.

prohibited by the computational expense. Hence in the following two sections, we discuss two discrete chaotic maps and provide analytical and numerical evidence of cutoffs in chaotic mixing.

\section{THE SUPER-EXPONENTIAL MIXING CURVE}

In this section we use a simple example to explain why we expect to see the concave mixing trajectory in the beginning of a chaotic mixing process. Consider the Baker's map on $T^{2}$ :

$$
S\left(x_{1}, x_{2}\right)= \begin{cases}\left(2 x_{1}, \frac{1}{2} x_{2}\right) \bmod 1, & \text { if } 0 \leq x_{1}<\frac{1}{2} \\ \left(2 x_{1}, \frac{1}{2}\left(x_{2}+1\right)\right) \bmod 1, & \text { if } \frac{1}{2} \leq x_{1}<1\end{cases}
$$
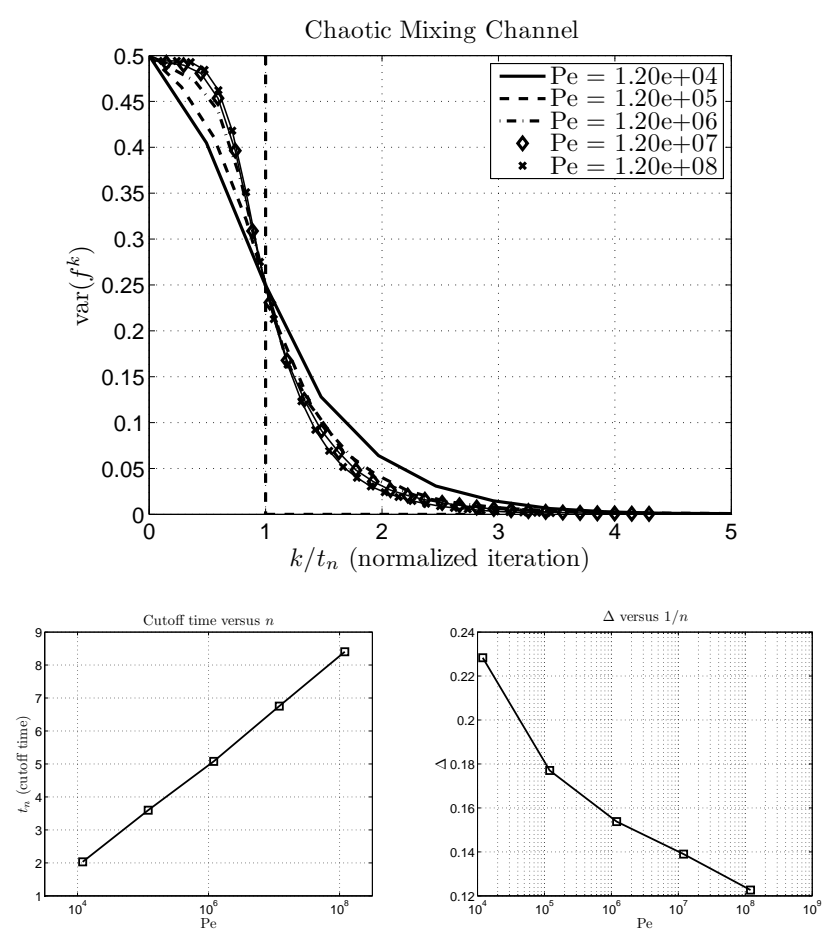

Figure 4. TOP: NORMALIZED TRAJECTORIES OF THE MICROFLUIDIC MIXING CHANNEL. BOTTOM LEFT: NEAR LINEAR RELATION BETWEEN CUTOFF TIME $t_{n}$ AND Pe. BOTTOM RIGHT: $\Delta_{\hat{B}}^{3}$ AS A FUNCTION OF Pe. THE DECREASE OF $\Delta_{\hat{B}}^{3}$ WITH $t_{n}$ SUGGESTS A CUTOFF.

with initial condition $f^{0}(x)=\sqrt{\pi} \cos \left(2 \pi x_{2}\right)$. The action of the Koopman operator on $f^{0}(x)$ is very simple: it doubles the frequency of the cosine wave at each iteration, as shown in Figure 6. We further apply a diffusion operator with diffusivity $D$ after every iteration to make the process diffusive. Just like in the mixing channel problem, we want to know how the variance changes with iteration number $k$. This problem is analytically solvable:

$$
f^{k}(x)=\sqrt{\pi} e^{-4 \pi^{2} D 2^{2 k}} \cos \left(2 \pi 2^{k} x_{2}\right) \text { for } k=1,2, \ldots
$$

and the variance of $\left(f^{k}(x)\right)$ is $\operatorname{var}\left(f^{k}(x)\right)=e^{-8 \pi^{2} D 2^{2 k}}$, a doubly exponential function of $k$. We plot $\operatorname{var}\left(f^{k}(x)\right)$ in Figure 7 with different $D$ values. This figure shows that the set of trajectories presents a cutoff with cutoff time $t_{n} \sim-\log (D)$. The trajectories we see in the mixing channel simulation have the same tendency as the Baker's Map simulation. In the next section we numerically simulate another discrete map, the Standard Map, to provide the additional numerical evidence of the cutoff in chaotic maps. 


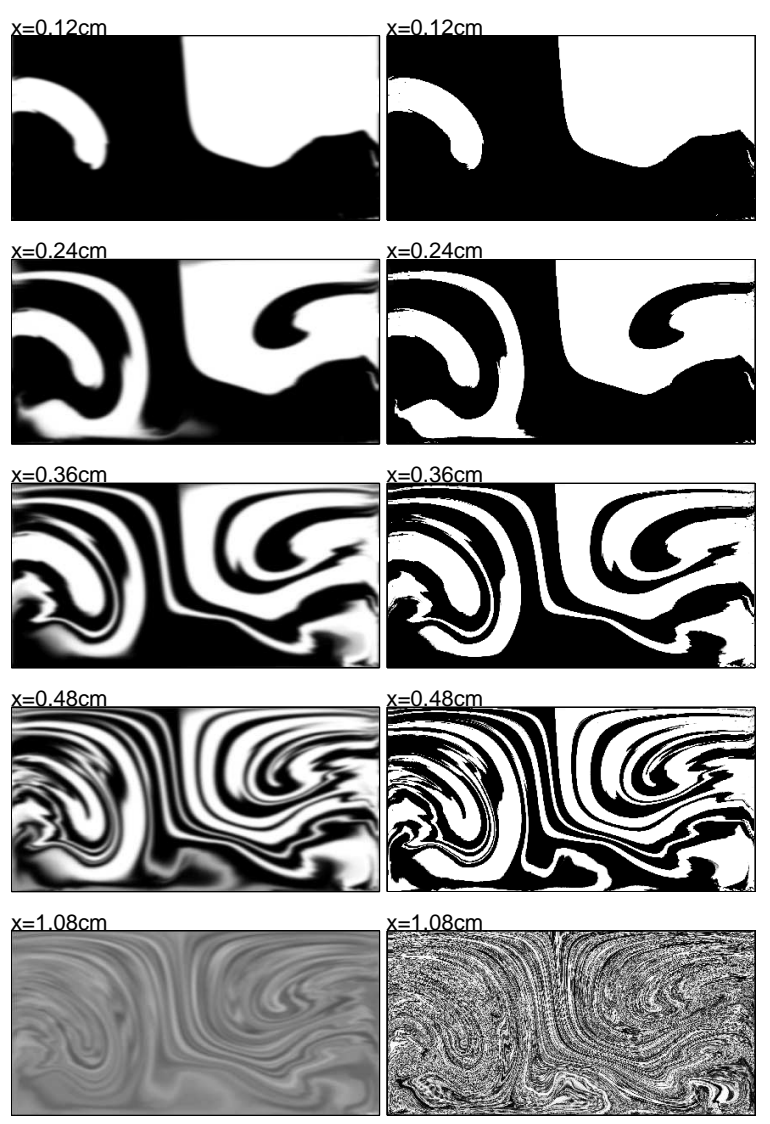

Figure 5. CHANNEL CROSS-SECTIONS AT THE END OF CYCLES 1, 2, 3, 4, 9 (TOP TO BOTTOM) FOR Pe $=1.2 \times 10^{6}$ (LEFT) AND $1.2 \times 10^{9}(\mathrm{RIGHT})$.
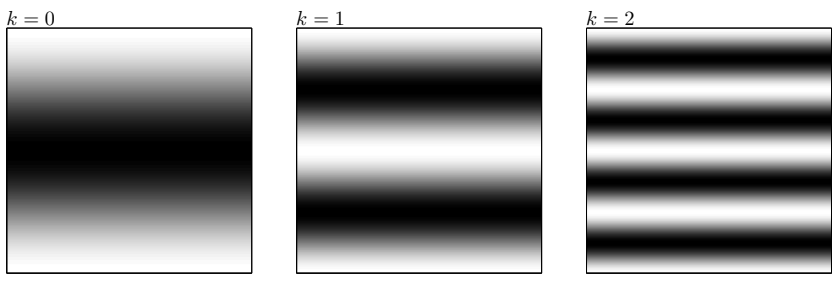

Figure 6. THE FIRST THREE ITERATIONS OF A FUNCTION $f^{0}=$ $\cos \left(2 \pi x_{2}\right)$ ADVECTED BY THE BAKER'S MAP. IT SIMPLY DOUBLES THE FREQUENCY OF THE COSINE FUNCTION IN THE $x_{2}$ DIRECTION.

\section{STANDARD MAP SIMULATION}

We study the Standard Map on $T^{2}$ :

$$
\begin{aligned}
& x_{1}^{\prime}=x_{1}+x_{2}+\varepsilon \sin 2 \pi x_{1}(\bmod 1), \\
& x_{2}^{\prime}=x_{2}+\varepsilon \sin 2 \pi x_{1}(\bmod 1) .
\end{aligned}
$$

This map is known to be chaotic for certain values of $\varepsilon$. Various studies of how a point is advected by the map can be found, for

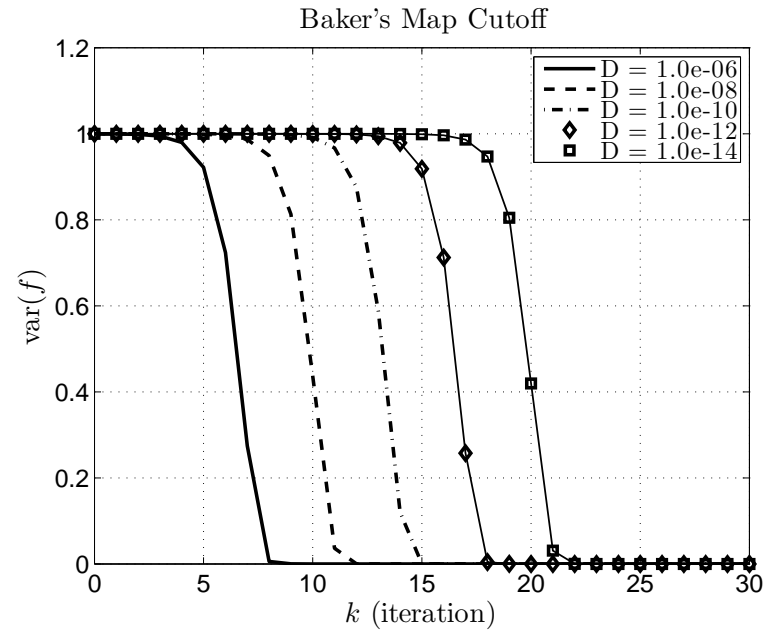

Figure 7. VARIANCE EVOLUTION OF A FUNCTION ADVECTED BY THE BAKER'S MAP, SHOWING CUTOFF.

example, in [23]. Here we mainly focus on how a scalar function is evolved by the map with additional small diffusion. Because this map is volume preserving, its invariant measure is uniform. We always scale the mean and standard deviations of $f^{0}$ to be 1. The Standard Map is known to have some non-chaotic region when $\varepsilon$ is not zero, so the distance $d$ converges to a value $m \neq 0$, which is why Definition 3 of cutoff is slightly modified from that in [10].

The evolution of the variance of $f_{n}^{k}$ with $n$ ranging from $2500^{2}$ to $80000^{2}$ using $B_{n}$ and $\bar{B}_{n}$ as the Koopman operators are shown in the left of Figures 9 and 10. The tendency is clear: as $n$ becomes larger, the variance stays high $\left(M_{B}=M_{\bar{B}}=1\right)$ for more iterations and then drops rapidly to $m_{B}=0.4521$ and $m_{\bar{B}}=0.4498$, respectively - they do not drop to zero because there are unmixed "islands". The rapid dropping region also becomes slightly longer when $n$ increases. To see whether this evolution presents a cutoff, we let $t_{n}$ be the time where each trajectory passes through $\left(M_{\star}+m_{\star}\right) / 2$, where $\star=\{B, \bar{B}\}$, and normalize all the trajectories by rescaling $t_{n}$ to 1 . The results are plotted in the right of Figures 9 and 10. Although the normalized trajectories are very similar, one can still see that when $n$ gets larger, the trajectory becomes sharper. In the upper plot of Figure 11 we plot $t_{n}$ versus $1 / n$ on a logarithmic scale and we see two straight lines. Note that for both cases, we have $D \sim O(1 / n)$. Hence this plot shows that the cutoff time is inversely proportional $\log (D)$. Just like we did in the mixing channel trajectories, we plot $\Delta_{B}^{3}$ and $\Delta_{\bar{B}}^{3}$ versus $t_{n}$ in the right of Figure 11, showing that when $t_{n}$ increases, both $\Delta_{B}^{3}$ and $\Delta_{\bar{B}}^{3}$ decay slightly sub-linearly, but clearly as $n$ grows, $\Delta_{B}^{3}$ and $\Delta_{\bar{B}}^{3}$ decrease, which strongly suggests that both sequences of Markov Chains present cutoffs. 


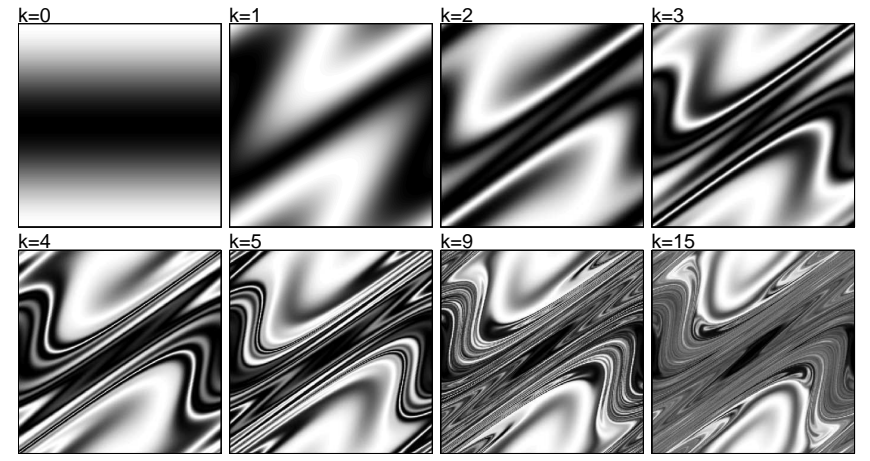

Figure 8. THE FIRST EIGHT ITERATIONS OF $f_{n}^{k}$ WHEN $f^{0}=$ $\cos \left(2 \pi x_{2}\right)$ IS ADVECTED BY THE STANDARD MAP WTIH $\varepsilon=0.3$ AND $n=500$.
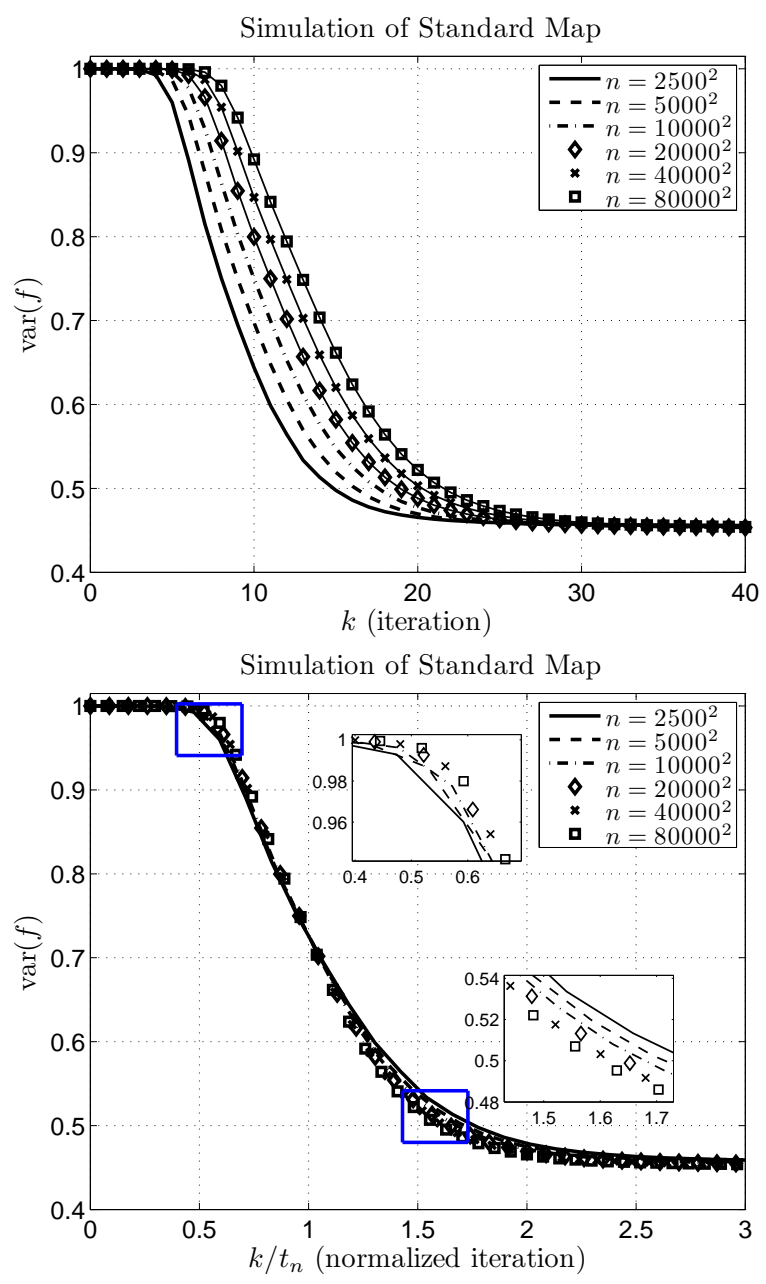

Figure 9. TOP: VARIANCE OF $f_{n}^{k}$ VERSUS ITERATION NUMBER FOR THE STANDARD MAP. WE USE $\varepsilon=0.3, f^{0}=\cos \left(2 \pi x_{2}\right)$, AND NUMBER OF GRID CELLS $n$ VARIES FROM $2500^{2}$ TO $80000^{2}$. BOTTOM: RESCALED VERSION OF THE TOP PLOT, SO THAT ALL TRAJECTORIES PASS THROUGH THE POINT $(1,0.7260)$. THE TWO SMALL PLOTS SHOW DETAILED VIEWS OF THE CORNERS.

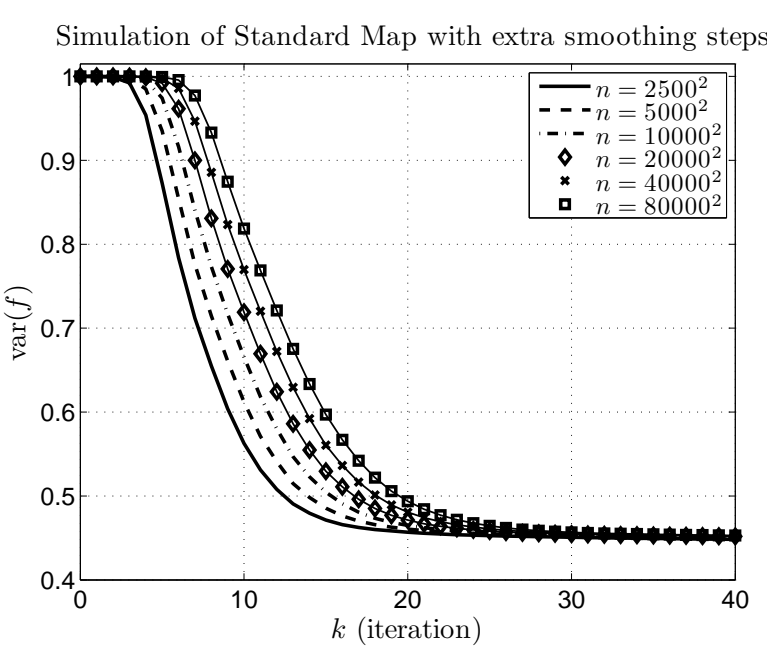

Simulation of Standard Map with extra smoothing steps

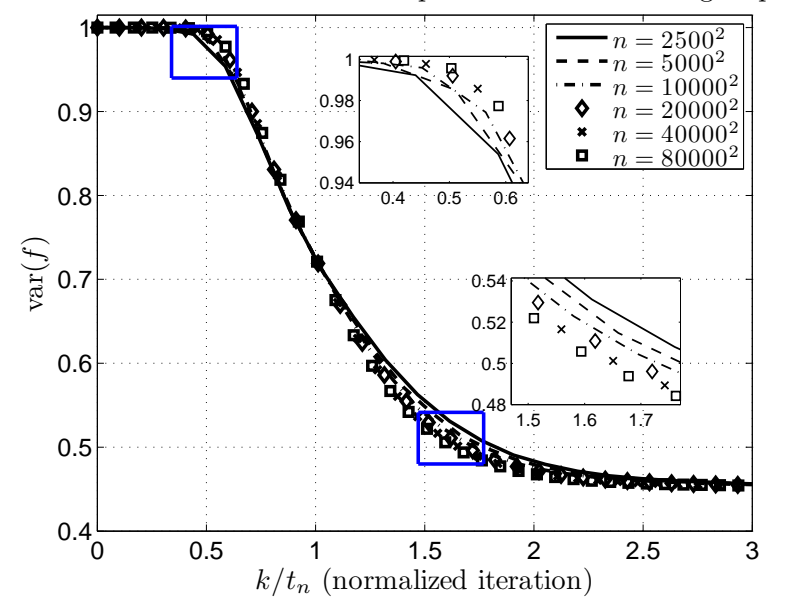

Figure 10. TOP: VARIANCE OF $f_{n}^{k}$ VERSUS ITERATION NUMBER FOR THE STANDARD MAP SIMULATION WHEN A SMOOTHING STEP IS ADDED AFTER EACH ITERATION. WE USE $\varepsilon=0.3, f^{0}=$ $\cos \left(2 \pi x_{2}\right)$, AND NUMBER OF GRID CELLS $n$ VARIES FROM $2500^{2}$ TO $80000^{2}$. BOTTOM: RESCALED VERSION OF THE TOP PLOT, SO THAT ALL TRAJECTORIES PASS THROUGH THE POINT $(1,0.7260)$. THE TWO SMALL PLOTS SHOW DETAILED VIEWS OF THE CORNERS.

\section{CONCLUSION}

We provide numerical evidence that mixing processes in a number of fluid and chaotic map systems present cutoffs in the sense of finite Markov Chains, in the limit of small diffusion. This means that as the diffusion tends to zero, the time over which the variance of an advected scalar function decreases significantly tends to zero, relative to the onset time of the decrease.

\section{REFERENCES}

[1] Ottino, J. M., and Wiggins, S., 2004. "Introduction: Mixing in microfluidics". Philosophical Transactions of the Royal Society A: Mathematical, Physical and Engineering 

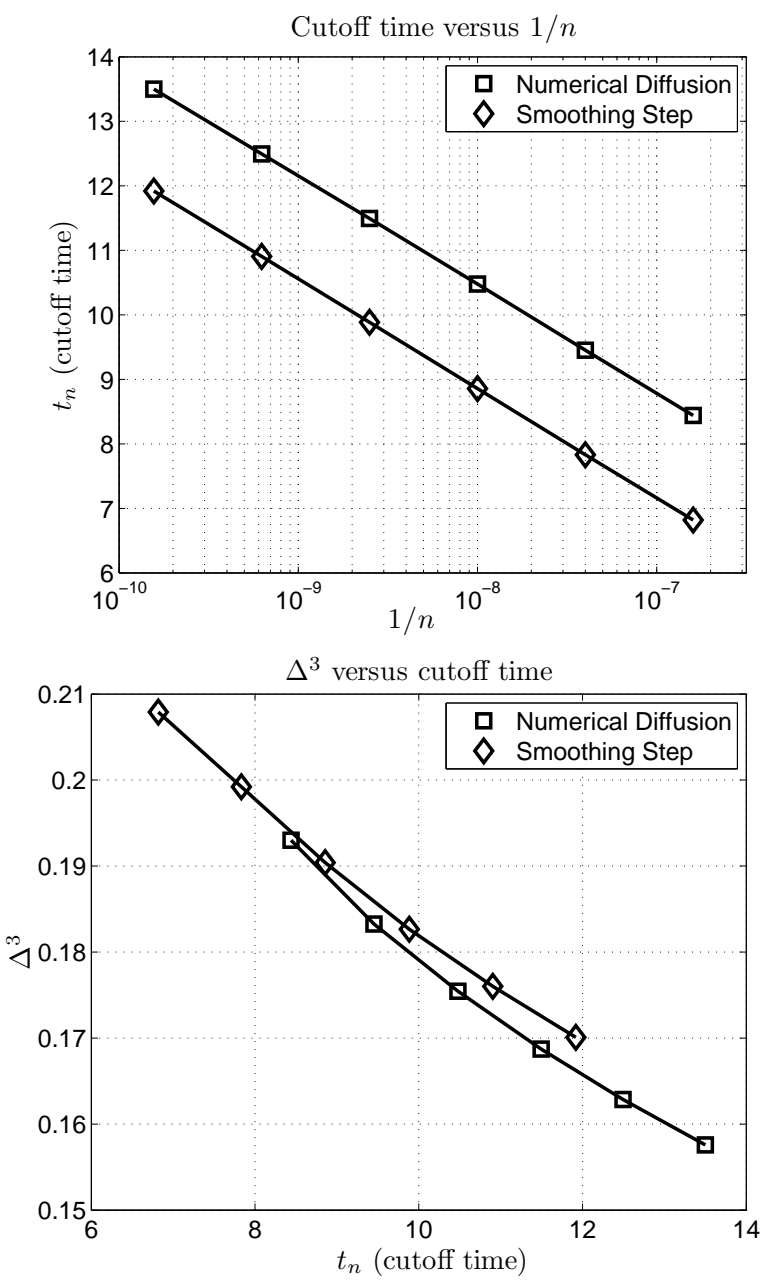

Figure 11. TOP: CUTOFF TIME $t_{n}$ RELATED TO $1 / n$ ON A LOGARITHMIC SCALE. SINCE WE HAVE $D \sim O(1 / n)$ FOR OUR SIMULATION STRATEGY, ONE CAN CONCLUDE THAT $t_{n}$ IS INVERSELY PROPORTIONAL TO $\log (D)$. BOTTOM: CONVERGENCE OF NORMALIZED TRAJECTORIES TO THEIR LIMITS. $\Delta^{3}$ IS DEFINED IN EQUATION (8). BOTH CURVES PREDICT THAT WHEN $t_{n}$ IS VERY LARGE, $\Delta^{3}$ GOES DOWN, AND THE NORMALIZED TRAJECTORY WOULD LIKELY LIMIT TO A STEP FUNCTION.

Sciences, 362(1818), March, pp. 923-935.

[2] Voth, G. A., Haller, G., and Gollub, J. P., 2002. "Experimental measurements of stretching fields in fluid mixing". Physical Review Letters, 88(25), June.

[3] Tsang, Y.-K., Antonsen, Jr., T. M., and Ott, E., 2005. "Exponential decay of chaotically advected passive scalars in the zero diffusivity limit". Physical Review E, 71, June, p. 066301.

[4] Thiffeault, J.-L., and Childress, S., 2003. "Chaotic mixing in a torus map". CHAOS, 13(2), June, pp. 502-507.

[5] Fereday, D. R., Haynes, P. H., Wonhas, A., and Vassilicos, J. C., 2002. "Scalar variance decay in chaotic advection and Batchelor-regime turbulence". Physical Review E, 65(3),
February, p. 035301.

[6] Mathew, G., Mezić, I., and Petzold, L., 2005. "A multiscale measure for mixing". Physica D, 211(1-2), November, pp. 23-46.

[7] Thiffeault, J.-L., 2004. "Scalar decay in chaotic mixing". In Transport in Geophysical Flows: Ten Years After, Proceedings of the Grand Combin Summer School.

[8] Haynes, P. H., and Vanneste, J., 2005. "What controls the decay of passive scalars in smooth flows?". Physics of Fluids, 17, September, p. 097103.

[9] Cerbelli, S., Adrover, A., and Giona, M., 2003. "Enhanced diffusion regimes in bounded chaotic flows". Physics Letters A, 312(5), June, pp. 355-362.

[10] Diaconis, P., and Saloff-Coste, L., 2006. "Separation cutoffs for death and birth chain". Annals of Applied Probability, 16(4), pp. 2098-2122.

[11] Pierrehumbert, R. T., 2000. "Lattice models of advectiondiffusion". Chaos, 10(1), March, pp. 61-74.

[12] Froyland, G., 2001. "Extracting dynamical behaviour via Markov models". In Nonlinear Dynamics and Statistics. Birkhäuser, Basel, pp. 283-324.

[13] Diaconis, P., Graham, R. L., and Morrison, J. A., 1990. "Asymptotic analysis of a random walk on a hypercube with many dimensions". Random Structures and Algorithms, 1(1), pp. 51-72.

[14] Yang, Z., Goto, H., Matsumoto, M., and Maeda, R., 2000. "Ultrasonic micromixer for microfluidic systems". In The Thirteenth Annual International Conference on Micro Electro Mechanical Systems, pp. 80-85.

[15] Aamo, O. M., Krstic, M., and Bewley, T. R., 2003. "Control of mixing by boundary feedback in 2D channel flow". Automatica, 39, pp. 1597-1606.

[16] Balogh, A., Aamo, O. M., and Krstic, M., 2005. "Optimal mixing enhancement in 3D pipe flow". IEEE Transactions on Control Systems Technology, 13, pp. 27-41.

[17] Aamo, O. M., and Krstic, M., 2004. "Feedback control of particle dispersion in bluff body wakes". International Journal of Control, 77, pp. 1001-1018.

[18] Yuan, C. C., Krstic, M., and Bewley, T., 2004. "Active control of jet mixing". In IEE Proceedings: Control Theory and Applications, Vol. 151, pp. 763-772.

[19] Aamo, O. M., and Krstic, M., 2002. Flow Control by Feedback. Springer.

[20] Stroock, A. D., Dertinger, S. K. W., Ajdari, A., Mezić, I., Stone, H. A., and Whitesides, G. M., 2002. "Chaotic mixer for microchannels". Science, 295(5555), January, pp. 647651.

[21] Ottino, J. M., and Wiggins, S., 2004. "Designing optimal micromixers". Science, 305(5683), July, pp. 485-486.

[22] Liang, T.-C., and West, M., 2008. Optimized mixing in microfluidic channels. (In preparation).

[23] Ott, E., 2002. Chaos in Dynamical Systems. Cambridge University Press. ISBN 0521010845. 